\title{
Gestión por proyecto y autonomía escolar. Contribuciones para un estado del arte sobre la cuestión*
}

\author{
María Cecilia Bocchio **
}

\section{Resumen}

El artículo que se presenta muestra el estado del arte construido en el marco de una investigación titulada "La difícil construcción de la autonomía escolar. Estudio del impacto del Proyecto de Promoción de la Autonomía Escolar (PPAE) ${ }^{1 .}{ }^{12}$ La investigación constituye una tesis de licenciatura realizada en el marco del proyecto "Estudios sobre Políticas Educativas: las regulaciones del Sistema Educativo y sus efectos en la gestión de las instituciones educativas y en el trabajo de los docentes en el nivel medio y superior universitario" (2010-2011) ${ }^{3}$.

Se incluyen en el estado de arte sistematizado los principales aportes teórico-metodológicos y conclusiones de la investigación desarrollada.

\footnotetext{
* Artículo recibido el 29 de mayo. Aceptado el 31 de julio.

En inglés: Management through projects and school autonomy. Contributions for an state of the art

** María Cecilia Bocchio es Licenciada en Ciencias de la Educación por la Universidad Nacional de Córdoba. Becaria Doctoral Erasmus-Mundus Universidade de Lisboa, Portugal. Doctorado en Educación. Mención en Política y Administración Educativa. Contacto: mcbocchio@gmail.com.ar

${ }^{1}$ En adelante PPAE.

${ }^{2}$ Trabajo Final de Licenciatura en Ciencias de la Educación.UNC. María Cecilia Bocchio, Carla Errico. Dirigido por la Mgter. Nora Lamfri. Titulado "La difícil construcción de la autonomía escolar". Estudio del Proyecto Promoción Autonomía de la Escuela (PPAE).

3 Proyecto dirigido por la Dra. Estela María Miranda y codirigido por la Mgter. Nora Lamfri, radicado en el Centro de Investigación María Saleme de Burnichon de Facultad de Filosofía y Humanidades, Universidad Nacional de Córdoba. (Subsidio SECyT UNC).
} 


\section{Palabras clave}

Estado del arte - autonomía escolar - gestión por proyectos.

\section{Abstract}

This paper presents the state of the art construed in the context of a research titled:"The difficult construction of school autonomy. Study of the impact of the Project for the Promotion of School Autonomy (PPAE) "The research, is a thesis involved in the project "Educational Policy Studies: Regulations of the Education System and its effects on the management of educational institutions and the work of teachers in secondary schools and university level". (2010-2011)

Is included in the state of art systematized, the main theoreticalmethodological contributions and conclusions of the research.

\section{Keywords}

State of art - school autonomy - management by project.

\section{Sobre la "necesidad" de construir un estado del arte}

El objetivo de este artículo es dar cuenta de aquello que ya fue "dicho" en materia de políticas de gestión por proyecto y autonomía escolar, profundizando en los estudios desarrollados en Argentina.

Se incluyen como contribución al estado del arte compilado los principales aportes en materia de contexto, texto y metodologías de investigación utilizadas para interpretar la implementación del PPAE desde las voces de directores de escuelas secundarias de la Ciudad de Córdoba, Argentina.

El Trabajo Final de Licenciatura en Ciencias de la Educación supuso la construcción de un estado del arte que con el avance de la investigación fue ampliando sus dimensiones de análisis, constituyéndose en una "caja de herramientas" fundamental para el desarrollo teórico-metodológico del estudio; apoyando la definición del objeto de estudio y la proposición de nuevas miradas sobre temáticas que envuelven la gestión escolar actual y que demandan ser problematizadas.

El objeto de estudio de la investigación fueron directores de escuelas de gestión pública que estuvieron implicados en la implementación del PPAE, procurando indagar particularmente en el impacto del proyecto en la gestión de las escuelas que participaron. 
Este artículo y el conjunto de investigaciones que se presentan se enmarcan en el análisis de nuevos instrumentos de regulación del Sistema Educativo. En Argentina, la gestión por proyecto y la autonomía escolar cobraron presencia en la política educativa nacional, principalmente a partir de la década del noventa, y constituyen discursos que al parecer "han llegado para quedarse" en el gestión escolar, corriendo graves riesgos de ser naturalizados como meras retóricas, dejando de ser cuestionados sus objetivos y los efectos que suscitan en los actores escolares.

\section{Estado del arte: texto y contexto de investigación}

Los criterios a partir de los cuales se organiza el estado del arte responden al contexto de producción de las investigaciones y a los ejes de análisis indagados por ellas.

Las principales temáticas abordadas en los estudios son dimensiones íntimamente relacionadas en el discurso y en los efectos de la Reforma Educativa. Entre ellas se destacan: la gestión escolar, la autonomía escolar, la evaluación de proyectos y las implicancias de las políticas educativas en el trabajo de los directivos. Estas divisiones son puramente analíticas, ya que a partir de las numerosas investigaciones recopiladas fue posible constatar la interrelación que existe entre las dimensiones mencionadas.

En el plano internacional, específicamente en el contexto europeo, se destacan las investigaciones de S. Ball (1994); J. Barroso (2003a) y J. C. Matos Riveiro (2007). Los autores concuerdan en señalar que existe una nueva forma de concebir a la educación a partir de orientaciones que provienen del campo económico, lo cual implica la definición de nuevos dispositivos de regulación que afectan a las instituciones educativas y a las funciones tradicionales de los directivos. Desde esta perspectiva, los directores escolares incorporan un nuevo lenguaje propio de la lógica técnicoburocrática y son concebidos como gerentes que se deben ocupar del uso eficiente de recursos, pasando a ser responsabilizados acerca de resultados escolares alcanzados por el alumnado.

S. Ball (1994) sostiene que los planes de estudio, el mercado, la gestión, la evaluación de docentes y alumnos son mecanismo de control que obligan a los agentes a la rendición de cuentas. Además, reconoce la existencia de un nuevo modo de gestión "tecnocrática", cuyas características principales son la impersonalidad, la especialización y la monopolización estratificada del conocimiento intelectual.

J. Barroso (2003a) afirma que a partir de la implementación de este tipo de políticas de corte neoliberal, se produce la emergencia de "multi-regulaciones" provenientes de distintos sectores que impactan en el sistema educativo y en la manera de gestionar a las instituciones. Resalta como principales problemáticas la proliferación de políticas educativas de países centrales que son aplicadas en contextos sociales, económicos y políticos diversos, legitimando así la recontextualización de programas y proyectos educativos que se aplican como "métodos" para alcanzar la autonomía escolar. 
J. C. Matos Riveiro (2007) reconoce a los directivos de escuelas como el principal objeto de las políticas educativas. Para el autor, las transformaciones en la gestión permiten percibir nuevos modos de regulación gerencial, en los cuales los directores acaban por transformarse en decodificadores de aquellos modos de regulación elaborados desde instancias centrales de gobierno.

Continuando con esta línea de análisis, en el contexto latinoamericano se destacan las investigaciones de N. Krawczyk (2002) E. Oto Shirmona y R. Campos (2006), M. Alves García, S. Barreto Anadon (2006), D. Andrade Oliveira (2007) y (2006) (en Feldfeber, M. y Andrade Oliveira, D. comps. 2006). Se contextualizan en las reformas educativas que acontecieron de forma masiva en la década del noventa en la región latinoamericana, reformas que con algunos rasgos específicos entre los países, legitimaron en líneas generales retóricas asociadas a la "modernización y profesionalización" del sistema educativo. Se introdujo para los autores un nuevo modo de regulación y gestión gerencial en el ámbito educativo que ha promovido, entre otros efectos, la intensificación del trabajo docente y el incremento potencial de la "colonización administrativa" del trabajo del director, que debe responder ahora a múltiples evaluaciones y auditorías externas.

En Argentina encontramos numerosas investigaciones que abordan los efectos de las reformas educativas de corte neoliberal en la gestión escolar, entre ellas: $\mathrm{N}$. Martínez de Pérez et ál. (2003); M. Manzione (2003); G. Salse (2003); A. Corrado Vázquez (2003); M. Macri, (2005); S. Kravetz (2004); A. Castro (2006) y M. Sendón (2007).

M. Manzione (2003) y G. Salse (2003) analizan el rol del director en la emergencia de estilos de gestión, lo cual conduce a indagar acerca de las concepciones de gestión escolar que prevalecen en los discursos de los directivos. Las autoras resaltan que cada institución genera un proceso singular, el cual conlleva forjar un estilo particular de gestión directiva.

N. Martínez de Pérez et ál. (2003) coinciden en afirmar que en el marco de la gestión descentralizada, la autonomía cobra relevancia en la formulación del Proyecto Educativo Institucional, transformándose este en el único instrumento de carácter colectivo que persiste en las escuelas, hecho que no significa que se realice un trabajo conjunto en su elaboración y reformulación. Esta problemática se agudiza en el nivel medio y superior no universitario, en los cuales las posibilidades de trabajo colectivo como producto de las condiciones de trabajo son escasas.

S. Kravetz (2004) investigó la Reforma Educativa en la década del 90, buscando interpretar sus principales líneas de acción y las particularidades de la implementación en el nivel medio de la provincia de Córdoba. Focalizó en el análisis de programas que respondieron a la lógica de gestión por proyecto, entre ellos el Programa Nacional de Becas Estudiantiles, el Programa de Descentralización y Mejoramiento de la Educación Media y los programas de Capacitación Docente en el marco de la Red. La autora sostiene que la modalidad de implementación de la reforma en Córdoba estuvo 
signada por la vertiginosidad de las acciones efectuadas, en las cuales primó una modalidad tecnocrática de imposición de innovaciones basada en el control de resultados, que derivó en el sostenimiento de un modelo burocrático de supervisión y control de las instituciones educativas.

M. Macri (2005) y M. Sendón (2007) cuestionan el vínculo unívoco entre autonomía y el mejoramiento de los resultados que obtienen las instituciones educativas. Afirman que la autonomía a nivel escolar requiere un proceso de reconocimiento de demandas históricas y la construcción de nuevo marco regulatorio que brinde más libertades a las escuelas.

A. Castro (2006) analiza la gestión como discurso y tecnología de regulación identificando la producción de un doble efecto: por un lado, posibilita el ordenamiento y optimización del funcionamiento de la escuela $y$, por otro, promueve en el discurso pedagógico la introducción de conceptos propios del ámbito empresarial.

También se ha encontrado una serie de trabajos centrados en la evaluación y análisis de proyectos, que impulsaron transformaciones en la gestión de las instituciones educativas. En Portugal, se destaca el aporte de J. Barroso (2003b), quien desde una perspectiva crítica elabora el concepto de "regulación institucional" para aludir a la intervención de las autoridades públicas en los modos de regular las escuelas resalta la coexistencia de medidas de refuerzo de la autonomía de las escuelas y de nuevos mecanismos de control. Sustentado en esta perspectiva discute la implementación de políticas para el combate de las desigualdades escolares, entre ellas el programa "Programa Educación para Todos". Califica este tipo de programas como políticas financieras que no permiten adaptarse al contexto escolar, pero que a la vez dan cuenta de la ausencia de una estrategia global superadora.

Desde otra perspectiva, más afín a la gestión por proyectos, R. R. Ramírez (2000) investigó en México los cambios que produjo en la gestión escolar la implementación del proyecto de innovación e investigación "La gestión en la escuela primaria". Este proyecto, suscripto al Acuerdo Nacional para la Modernización de la Educación Básica, buscó aumentar la calidad y la equidad de los servicios educativos. El autor concluye que a través de esta vía los actores escolares, principalmente los directivos, adquirieron herramientas para evaluar la situación de su plantel escolar y diseñar un plan de acción colectivo para superar las deficiencias encontradas.

En Argentina, M. Schneckenberg (2003) investigó la implementación de un proyecto denominado "Programa de Expansión, Mejora, e Innovación de la Enseñanza Media" (PROEM). Se implementó en Paraná en 1998 y se llevó a cabo con fondos del Banco Interamericano de Desarrollo (BID). El objetivo de dicho programa fue reformar la enseñanza media, priorizando y fortaleciendo la educación general, como base para la formación profesional específica o para la continuidad de los estudios en el nivel superior. La autora, teniendo en cuenta todos los actores involucrados en el contexto escolar, buscó analizar la "reacción" de la gestión de la escuela en la práctica cotidiana 
frente al establecimiento de una política de Reforma Educativa de origen centralizado. Entre las principales conclusiones se destaca que la construcción de un plan de reforma requiere, de parte de todos los actores que componen el cuerpo de la escuela, especialmente del gestor escolar, una actitud de reflexión colectiva permanente para que el cambio sea viable. La tarea del gestor, para la autora, implica llevar adelante una "osadía colectiva", que se traduce en la práctica cotidiana construida y reconstruida, dentro y fuera de la escuela. Finalmente sostiene que debe existir una relación dialéctica entre el Estado y la escuela, y entre esta última y la comunidad, para que la política tome el camino previsto.

I. Aguerrondo (2007) investigó el "Programa Escuelas por el Cambio", combinando la reflexión teórica y el análisis empírico de experiencias concretas que fueron promovidas por el proyecto. Dicho programa surge como una iniciativa desarrollada y ejecutada por la Fundación Compromiso y aplicada en 207 escuelas de gestión estatal ubicadas en diferentes puntos de la Argentina, durante el año 2000. El objetivo fundamental del programa fue la adquisición por parte de directores y maestros de capacidades fundamentales para definir las metas, los objetivos y el rumbo institucional de sus escuelas, e incorporar a su trabajo cotidiano las herramientas de gestión del programa. Estudió el impacto y los efectos del programa, abordando sus diferentes componentes. Entre los principales resultados se destaca la mejora en la calidad de la comunicación dentro de las instituciones, lo cual repercutió positivamente en el momento de tomar decisiones. Se resalta que las escuelas con equipos directivos estables lograron modificar su perspectiva acerca de la gestión y fundamentalmente apropiarse del proyecto, generando cambios durables en la gestión.

En términos generales, todos los trabajos realizaron un análisis de las políticas formuladas durante la década de los 90, de su adecuación a nivel mesopolítico y finalmente de cómo esas medidas afectaron a las instituciones educativas y sus actores; señalando (mayoritariamente) el distanciamiento que existe entre el ámbito de formulación de las políticas educativas y el de ejecución.

Las investigaciones muestran una tendencia general que apunta a la redefinición de la organización y gestión de los sistemas educativos, aludiendo al carácter paradójico que posee este proceso en el que al tiempo que se otorga una aparente mayor autonomía a las instituciones educativas, se crean mecanismos de responsabilidad y rendimiento de cuentas que deterioran el trabajo docente e influyen decisivamente en la gestión directiva.

Los autores sostienen la necesidad de elaborar desde instancias centrales estrategias alternativas que brinden a las instituciones educativas márgenes reales de autonomía en la gestión escolar, contribuyendo de este modo a superar las falacias que los discursos promueven y que las prácticas escolares demuestran.

\section{Aportes de la investigación desarrollada al estado del arte sobre la cuestión}




\section{Introducción al objeto de estudio de la investigación}

EI PPAE fue implementado entre los años 2003 y 2006 en Córdoba, en el marco del Programa de Modernización de la Provincia de Córdoba

Esta iniciativa implicó la utilización de una estrategia de focalización de recursos financieros, materiales, de asistencia técnica y una modalidad de ejecución novedosa para las escuelas: acuerdo explícito de la institución para participar mediante la firma de un "acta de compromiso", "catálogo de ofertas" de capacitación docente, "feria de editoriales y librerías" y "catálogo" de libros, etc.

El proyecto incluyó a 1362 escuelas en toda la provincia, de las cuales 384 correspondieron al nivel medio. Promovió la dotación de diferentes recursos orientados al fortalecimiento de la "gestión autónoma" de las escuelas participantes.

Así, las líneas de acción definidas por el proyecto fueron las siguientes: a) Desarrollo de Técnicas de Gestión Escolar; b) Dotación y Utilización de Recursos Didácticos y c) Acrecentamiento de Estrategias Pedagógicas y Didácticas.

Entre los objetivos del PPAE se pretendía que docentes y directivos elaboren propuestas de enseñanza y proyectos de retención escolar acordes con las necesidades socioeducativas de la población a la que atendían, proyectos que debieran repercutir en los índices de promoción escolar.

\section{Abordaje metodológico de la investigación}

El estudio desarrollado tuvo como propósito principal reconstruir y analizar el impacto del PPAE en la gestión directiva de escuelas de nivel medio de la ciudad de Córdoba.

La investigación desarrollada responde a un enfoque cualitativo, siendo un estudio de caso de carácter exploratorio, desde el cual se analizó la implementación del PPAE en nueve instituciones educativas de gestión pública de nivel medio de enseñanza.

El total de escuelas que participaron del PPAE, en la provincia de Córdoba, fue de 229 instituciones educativas que atendían a una matrícula en situación de vulnerabilidad socioeducativa, de las cuales 45 se localizaban en la ciudad de Córdoba. Establecimos contacto con todas ellas y en veintitrés el equipo directivo no era el mismo que estaba en el momento de la implementación del PPAE. En trece escuelas fue recurrente la falta de predisposición de los directores para ser entrevistados, argumentando principalmente la carencia de tiempo y la necesidad de dar respuestas a las demandas de la escuela. Ante estas situaciones el universo de estudio de investigación se conformó por nueve escuelas.

La estrategia metodológica implicó dos tipos de fuentes de datos: documentales (normas legales, documentos oficiales del proyecto, informes de evaluación, auditorías, etc.) y entrevistas semiestructuradas a distintos actores.

Los directivos entrevistados fueron quienes participaron de la implementación del PPAE y a la vez continuaron desempeñando funciones en los equipos directivos hasta 
2010, cuando las entrevistas fueron realizadas. Asimismo, consideramos como informantes clave a supervisores y funcionarios del Ministerio de Educación de la provincia de Córdoba, quienes fueron responsables de la planificación, implementación y evaluación del proyecto en cuestión.

En función de las demandas analíticas del objeto de estudio, se construyó una matriz de análisis siguiendo, principalmente, los aportes de M. Sendón (2007), estructurada a partir de tres ejes centrales: 1) Modelos de gestión directiva, 2) Estrategias de gestión directiva y 3) Sentidos atribuidos a la autonomía escolar.

\section{Principales conclusiones}

El análisis de los documentos oficiales y el contraste con el trabajo de campo realizado condujo a reconocer al director como un actor central en la implementación del PPAE. Se desempeñó como decodificador de esta política educativa y operó como responsable del proceso de implementación de las diferentes líneas de acción que conformaron el proyecto.

Es posible sostener que las estrategias de gestión escolar desarrolladas por los directivos resultaron del interés pragmático que prevaleció en el momento de ejecución del PPAE, es decir, de los objetivos y beneficios que la participación en el proyecto podría generar para las escuelas.

El interrogante en términos de qué impacto generó en la gestión directiva el PPAE se articula con las estrategias utilizadas por los equipos directivos para su implementación. A fines analíticos se diferenciaron tres:

1. Estrategia de Oportunidad: la decisión del directivo de participar en el PPAE respondió al objetivo de obtener recursos para la escuela. Desarrollando, en algunos casos, proyectos de enseñanza y retención escolar que acabaron junto con el financiamiento del proyecto.

2. Estrategia de Continuidad: fue desplegada por directores de escuelas que utilizaron el PPAE como una herramienta para obtener recursos económicos que les posibilitaron financiar proyectos educativos que ya se estaban implementando. Se encuadran en este grupo escuelas cuyos Proyectos Educativos Institucionales se encontraban institucionalizados, como producto de una trayectoria institucional de trabajo conjunto entre docentes y directivos.

3. Estrategia de Iniciación: desarrollada por aquellas gestiones directivas que en el momento de implementarse el PPAE, se caracterizaban por la reciente incorporación del director en dicha función, cuerpos docentes pocos estables y un Proyecto Educativo Institucional en "estado de construcción".

Al parecer, las condiciones particulares que atravesaban a cada institución en el momento de implementarse el proyecto contribuyeron a otorgar un determinado sentido al PPAE y a la autonomía escolar. A través de las estrategias de gestión que los 
directivos utilizaron para implementar el PPAE fue posible reconocer la configuración de un modelo de gestión híbrido (Viñao Frago, 2002).

Viñao Frago (2002) diferencia cuatro modelos de gestión escolar definidos históricamente: 1) el modelo tradicional, en el que el director es un agente territorial que representa la administración educativa; 2) el modelo participativo democrático, el cual considera al director como un representante de la comunidad educativa ante la administración y la sociedad; 3) el modelo de liderazgo pedagógico, caracterizado por el auge de la cultura de la autonomía y la evaluación, buscando articular el proyecto educativo institucional con un contexto relativamente descentralizado y autónomo.

Por efectos de las multirregulaciones que atraviesan los sistemas educativos (Barroso, 2003a) y principalmente de las presiones del mercado en la definición de políticas educativas, los modelos de gestión mencionados acaban por articularse con el cuarto modelo: el neoliberal. En él el director opera como manager o gerente de una empresa que gestiona la escuela con escasos recursos, procura obtener diferentes fuentes de financiamiento y compite con otros centros educativos con su proyecto educativo institucional. Así, la regulación híbrida se manifiesta como un conjunto diverso de concepciones de dirección sedimentadas en el tiempo para explicar la multiplicidad de papeles a los que los directivos deben responder.

En este sentido, y partiendo de asimilar la gestión por proyecto ministerial, como el PPAE, a una estrategia de regulación que provee a las escuelas de gestión pública recursos materiales y económicos, es factible asociar el proyecto investigado a un mecanismo de financiamiento estatal focalizado que demanda al director la firma de un "contrato/acta de compromiso" para participar, respondiendo así a discursos y prácticas afines a lógicas gerenciales en la gestión escolar.

Diversas categorías de análisis construidas para el estudio de la gestión directiva en "contexto PPAE" responden a las influencias del modelo neoliberal. Entre ellas, la decisión de participar en el proyecto, la importancia del trabajo en equipo, la búsqueda y gestión de recursos en el mercado, la concepción del director como un gerente y la sobrecarga administrativa de la gestión del proyecto.

Las categorías antes mencionadas se articulan en los discursos y prácticas de los directivos con elementos afines a los modelos de dirección escolar definidos por Viñao Frago (2002), manifestando el carácter híbrido de adquiere la dirección escolar en contexto de nuevas regulaciones institucionales, como la gestión por proyecto.

En el título de esta investigación fue referenciada "la difícil construcción de la autonomía escolar". Para directivos y actores oficiales, la autonomía escolar en el marco del PPAE se vinculó fundamentalmente con la posibilidad de seleccionar recursos, en tanto la transmisión de herramientas de gestión que el proyecto pretendió difundir se redujo a técnicas de monitoreo y evaluación de resultados que debieron ser aplicadas estandarizadamente. 
Son estas técnicas insuficientes para dar respuesta a la "transmisión de un modelo de gestión con autonomía"; se manifiesta una visión reducida e instrumental de la gestión escolar, que "debe aplicar técnicas gerenciales" para obtener autonomía.

El análisis del proceso de recontextualización del PPAE en las escuelas dio cuenta de los modelos de gestión directiva institucionalizados, que viabilizaron aprovechamientos diferentes de los recursos provistos, tornando visible el nivel de ficción que pueden encubrir aquellos discursos que pretenden transmitir autonomía por medio de proyectos de escasa duración.

Desde la concepción de autonomía escolar que J. Barroso (2004) reconoce como la consecuencia de un proceso de construcción que acontece histórica e institucionalmente, es decir, como el producto de la elaboración que la gestión escolar y que particularmente la gestión directiva realiza no como un fin sí mismo, sino como un medio a disposición de las escuelas que demanda la creación de condiciones para que cada institución pueda desarrollar su autonomía, es factible afirmar que la gestión de la autonomía provista por el PPAE es consecuencia de la "gestión de autonomía" que cada director construyó como consecuencia de modelos de gestión directiva históricamente construidos, modelos que no dependen de la autonomía decretada por el PPAE y que por tanto anteceden y preceden la implementación del proyecto investigado.

\section{BIBLIOGRAFÍA}

Aguerrondo, I. (2007). Escuelas por el cambio: un aporte para la gestión escolar. Ed. Fundación Compromiso. IIPE-Unesco .Buenos Aires. Argentina.

Alves García M. \& Barreto Anadon S. (2006) en Feldfeber, M \& Andrade Oliveira, D comps. (2006), Políticas educativas y trabajo docente Nuevas regulaciones. ¿Nuevos sujetos? Ediciones Novedades Educativas .Argentina

Andrade Oliveira, D. (2007). La nueva regulación educativa en América Latina a nivel de las escuelas y de los docentes. Disponible en www.reseau-ameriquelatine.fr/ceisal.../PENS-EDU-1-ANDRADE.pdf (consultado 29/05/2013)

Ball, S. (1994). Las políticas educativas, las relaciones de poder y la labor docente. Education Reform: A Critical and Post-structural Approach. Open University Press, London.

Barroso J. (2011) Direcção de escolas e regulação das políticas: em busca do unicórnio. En Costa, J. A. e Neto-Mendes, A. orgs. (2011). A emergência do director da escola: questões políticas e organizacionais. Aveiro: Universidade de Aveiro.

Barroso, J. (1997). Autonomia e gestão da escola. Ministerio da Educação Portugal.

Barroso, J. (2003 a). Escuela pública: regulación, desregulación y privatización. Educación unisinios vol 8. N. ${ }^{\circ} 15$ (julio/diciembre 2004). 
Barroso, J. (2003 b). Organização e regulação dos ensinos básico e secundário, em Portugal: sentidos de uma evolução. Educ. Soc., Apr. vol. 24, no. 82, p. 63-92.

Barroso, J. (2004). Autonomía escolar: una ficción necesaria. Revista portuguesa de educacion Vol. 17, N 002. Universidad de Minho Braga. Pp. 49-83.

Castro A. (2006). La gestión escolar en cuestión. En Cuadernos de Educación. Publicación del área de educación de Centro de Investigaciones María Saleme de Burnichon FFYH UNC. Año 3 N. , 4. Págs. 225-233. Córdoba, Argentina.

Corrado Vázquez, A. (2003). Los sentidos de la autonomía institucional. Un estudio de casos en el conurbano bonaerense. En: Coloquio Nacional a diez años de la ley Federal de Educación ¿Mejor educación para todos? Córdoba, Argentina, 26, 27 y 28 de junio de 2003. Publicado en CD.

E. Oto Shirmona y R. Campos (2006) en Feldfeber, M \& Andrade Oliveira, D comps. (2006), Políticas educativas y trabajo docente Nuevas regulaciones. ¿Nuevos sujetos? Ediciones Novedades Educativas. Argentina.

Feldfeber, M. \& Andrade Oliveira, D. comps. (2006) Políticas educativas y trabajo docente Nuevas regulaciones. ¿Nuevos sujetos? Ediciones Novedades Educativas .Argentina.

Kravetz, S (2004). Contexto y texto de la reforma Educativa en la Provincia de Córdoba. Tesis de Maestría en Investigación Educativa. CEA. Córdoba, Argentina.

Macri, M. (2005). Descentralización educativa y autonomía institucional ¿Constituye la descentralización un proceso abierto hacia la autonomía de las escuelas públicas de Buenos Aires? Revista Iberoamericana de Educación N. ${ }^{\circ} 44$. Disponible en http://www.rieoei.org/deloslectores/242Macri.PDF (consultado 9/05/13)

Manzione M. A. (2003). Estilos de gestión de la institución escolar: una aproximación diagnóstica. En: Coloquio Nacional a diez años de la ley Federal de Educación ¿Mejor educación para todos? Córdoba, Argentina, 26, 27 y 28 de junio de 2003. Publicado EN CD.

Martínez de Pérez, N. y otros (2003). La reforma educativa y su incidencia en el modo de gestionar las escuelas de nivel medio: algunos indicadores puntuales. En: Coloquio Nacional a diez años de la Ley Federal de Educación ¿Mejor educación para todos? Córdoba, Argentina, 26, 27 y 28 de junio de 2003. Publicado en CD.

Matos Riveiro, J. C. (2007). La construcción el papel de los directores de escuela en la regulación local de las políticas públicas de educación. Sísifo: Revista de Ciências da Educação, N. $\quad 4$ págs. 59-66. Disponible en: http://dialnet.unirioja.es/servlet/oaiart?codigo=2512244 (Consultado 23/2/09)

Ramírez, R. R. (2000) La transformación de la gestión escolar: factor clave para mejorar la calidad de la educación. En Memoria del que hacer educativo 1995-2000, tomo I (pp. 173-192). México.

Salse, G. (2003). Los impactos de la reforma de la gestión administrativa en el marco de la implementación de la Ley Federal Educativa en el Ministerio de Cultura y 
Educación de Formosa. Coloquio Nacional a diez años de la ley Federal de Educación ¿Mejor educación para todos? Córdoba, Argentina, 26, 27 y 28 de junio de 2003. Publicado en CD.

Schneckenberg, M (2003). La implementación del PROEM (Programa Expansión, Mejora e Innovación en la Enseñanza Media) Como política educativa en la práctica de la gestión escolar. IIPE - UNESCO - Sede Regional Buenos Aires. Argentina. Disponible en: $\quad$ http://www.inep.gov.br/pesquisa/bbe-online/det.asp?cod=52436\&type $=\mathrm{M}$ (consultado 7/05/2013).

Sendón. M. (2007). Diferentes dimensiones de la autonomía de la gestión escolar: un estudio de casos en escuelas pobres de la Ciudad de Buenos Aires. Revista Iberoamericana de Educación 7 N. ${ }^{\circ}$ 44/2. Disponible en http://www.rieoei.org/deloslectores/1832Sendon.pdf (consultado 7/5/2013)

Tiramonti, G. (1996). Los Nuevos Modelos de Gestión Educativa y su incidencia sobre la Calidad de la Educación. FLACSO/ Serie Documentos e Informes de Investigación No 211. Buenos Aires, Argentina.

Viñao Frago, A. (2002). La cultura de las reforma escolares. En Perspectivas Docentes. N. ${ }^{\circ} 26, \mathrm{p} 38-56$. 